\title{
Comparison of Molecular, Clinicopathological, and Pedigree Differences Between Lynch-like and Lynch Syndromes
}

\section{Yun Xu}

Fudan University Shanghai Cancer Center

Cong Li

Fudan University Shanghai Cancer Center

Tian'an Guo

Fudan University Shanghai Cancer Center

\section{Yuqin Zhang}

Fudan University Shanghai Cancer Center

\section{Fangqi Liu}

Fudan University Shanghai Cancer Center

\section{YeXu( $\nabla$ yexu@shmu.edu.cn)}

Fudan University Shanghai Cancer Center

\section{Research article}

Keywords: colorectal cancer, Lynch syndrome, Lynch-like syndrome, pedigree, DNA mismatch repair

Posted Date: July 8th, 2020

DOI: https://doi.org/10.21203/rs.3.rs-35647/v1

License: () (1) This work is licensed under a Creative Commons Attribution 4.0 International License. Read Full License

Version of Record: A version of this preprint was published at Frontiers in Genetics on August 19th, 2020. See the published version at https://doi.org/10.3389/fgene.2020.00991. 


\section{Abstract}

Background: In this study, we compared the molecular, clinical, and pathological characteristics, as well as pedigrees, between patients with Lynch-like syndrome (LLS) and confirmed Lynch syndrome (LS) to develop appropriate management strategies for patients with LLS and their affected family members.

Methods: Between June 2008 and September 2018, 81 patients with LLS and 47 patients with LS who developed colorectal cancer (CRC) were enrolled in this study. Multigene panel testing included 139 genes and was performed for all patients. The variants identified in each group were described, and clinicopathological characteristics and pedigrees were compared between the two groups.

Results: In the LLS group, a total of 52 variants were detected in 44 (54.3\%) patients. Among the 52 variants, 17 were variants of unknown significance in mismatch repair genes, and the other most frequently mutated genes were MUYTH, POLE, BRCA2, and GJB2. The proportion of early-onset patients was significantly higher among the LS probands than among the LLS probands $\left(74.5 \%\right.$ and $53.1 \%$, respectively; $\chi^{2}=5.712, P$ $=0.017)$. On the other hand, the proportion of primary CRC developed in the rectum was higher in the LLS group than in the LS group $\left(25.9 \%\right.$ and $10.6 \%$, respectively; $\left.\chi^{2}=2.358, P=0.046\right)$. There were no significant differences in the occurrence of metachronous CRC $(P=0.632)$ and extra-CRC $(P=0.145)$ between the two groups. However, analysis of pedigrees showed that more patients developed CRC in the LS families $(P=$ 0.013), whereas more patients with extra-CRC were observed in the LLS families $(P=0.045)$. A higher prevalence of male patients was observed in the LLS families $(P=0.036)$.

Conclusions: LLS should be classified as a mixed entity, containing cases of true LS, other hereditary cancer syndromes, and sporadic CRC. The high risks of CRC and extra-CRC, found in our study, suggest that aggressive surgical management should be performed for LLS probands. In addition, we recommend a stringent surveillance protocol that should involve regular follow-ups of the intestine and other organs for probands and affected family members.

\section{Background}

Lynch syndrome (LS) is an autosomal dominantly inherited predisposition to colorectal cancer (CRC), accounting for approximately $2-5 \%$ of all CRC cases $[1,2]$. The consequent tumors present the phenotypes of mismatch repair $(M M R)$ protein deficiency and microsatellite instability (MSI). However, there is a lack of information on pathogenic variants (PVs) in $M M R$ genes for up to $50-70 \%$ of patients with $M M R$-deficient CRC tumors who were identified in population-based studies $[1,3]$. The majority of cases in this subset are characterized by hypermethylation of the $M L H 1$ promoter, which is also observed in approximately $15 \%$ of sporadic CRC cases [4, 5]. Variants in the BRAF oncogene are able to distinguish LS from sporadic $M M R$ deficient CRC; this has been demonstrated to be a powerful method for screening patients with LS [6, 7]. A subset of patients with CRC, who manifest the MMR deficiency but have no identified germline variant in either MMR genes or the BRAF gene (absence of MLH1 methylation), have been defined as having Lynchlike syndrome (LLS) $[1,3,8]$. It has been reported that LLS may account for up to $70 \%$ of clinically suspected LS cases with a high MSI and an MMR-deficient profile [9]. 
Molecular etiology of LLS still remains unknown, although previous findings have revealed that some groups of patients with LLS may be a mixture of true LS cases, with non-detected germline variants, and sporadic CRC cases $[8,10]$. Some researchers found that the risk of CRC was lower in families with LLS than in those with genetically confirmed LS $[10,11]$, while the age of CRC onset was similar for both diseases $[12,13]$. Nevertheless, the inability to determine the etiology of LLS hampers the development of effective screening and management policies for patients with LLS and the implementation of surveillance recommendations for these individuals and their affected relatives.

In the last decade, a wider application of multigene panel tests has provided more accurate molecular evidence for the diagnosis of LS; in the meantime, a considerable number of patients with LLS were identified at our center. Even though the genetic etiology of LLS is not defined, analyses of molecular, clinical, and pathological characteristics, as well as pedigrees of patients, may help guide decision making regarding surgical management, surveillance, and other interventions to reduce the future risks of cancer. This study was undertaken to compare the features of LS and LLS at the largest hereditary CRC research center of China, which could provide more information for the comprehensive understanding of LLS and guide management decisions for LS and LLS patients.

\section{Methods}

\section{Patients}

Between June 2008 and September 2018, a total of 139 patients with suspected LS and MMR-deficient profiles underwent curative surgeries, depending on the location of tumors, at the Fudan University Shanghai Cancer Centre. Multigene panel testing that included 139 genes was performed for all patients and some of their affected relatives. Informed consent for genetic analyses was obtained from all the patients. For patients with MMR deficiency variants in the $M L H 1$ or $M L H 1$ and PMS2 genes, detection of $B R A F$ V600 variants was performed to exclude sporadic CRC.

The inclusion criteria of our study were as follows: (a) CRC confirmed by post-operative pathology; (b) $M M R$ deficiency confirmed by immunohistochemistry; (c) the wild-type BRAF V600 variant confirmed in patients without PVs in MMR genes. A total of 128 patients who met the inclusion criteria were enrolled in this study. Among these, 47 (36.7\%) patients who were found to carry PVs in MMR genes were classified into the LS group, and 81 (63.3\%) patients without PVs in MMR genes and without BRAF V600 variants were classified into the LLS group. Carriers of variants of unknown significance (VUS) in MMR genes were also classified into the LLS group. Eleven patients without PVs in MMR genes but carrying a BRAF variant were excluded.

\section{Data Collection and Follow-up}

For the 128 enrolled patients, the demographic information, pathological results, and tumor histories were retrospectively collected. The pedigrees of their families were obtained through interviews of patients and their first- and second-degree relatives, including children, siblings, parents, grandparents, aunts, and uncles. The patient and each relative were asked to report whether the relative had ever been diagnosed with cancer. 
For each relative, the sex of the patient, type of cancer, and age at diagnosis were recorded. Pathology documentation of cancers among relatives was systematically collected, if available.

Follow-ups were conducted for all recruited patients every 2-3 months. During the follow-up evaluation, the occurrence of metachronous CRC, distant metastases, and extra-CRC was recorded. Treatment options for these events were formulated based on the recommendations of our multidisciplinary team. Meanwhile, new cases of tumors in their families were noted, and next-generation sequencing (NGS) was recommended for these patients. This study was censored on May 30, 2020.

\section{Next-generation sequencing}

Peripheral blood $(10 \mathrm{~mL})$ was collected, stored in ethylenediaminetetraacetic acid tubes, and allowed to stand at $25^{\circ} \mathrm{C}$ for $2 \mathrm{~h}$. The supernatant was transferred to a $15-\mathrm{mL}$ centrifuge tube and then centrifuged for $10 \mathrm{~min}$ at $2200 \mathrm{~g}$ at $4^{\circ} \mathrm{C}$. Thereafter, the intermediate white blood cells were transferred to a $1.5-\mathrm{ml}$ centrifuge tube. The DNA was recovered using the MagPure FFPE DNA LQ Kit (Magen). NGS was conducted on the germline DNA as a standard genetic testing for germline analysis.

DNA quantification was performed using the Qubit 2.0 Fluorimeter with the dsDNA HS assay kits (Life Technologies, Carlsbad, CA). A minimum of $50 \mathrm{ng}$ of DNA was required for NGS library construction. DNA shearing was performed using Covaris M220, followed by end repair, phosphorylation, and adaptor ligation. Fragments measuring 200-400 bp were selected using AMPure beads (Agencourt AMPure XP Kit), followed by hybridization with capture probes baits, hybrid selection with magnetic beads, and PCR amplification. The quality and size range of amplified fragments were then assessed by performing bioanalyzer highsensitivity DNA assay. Paired-end sequencing of the indexed samples was performed on a NextSeq 500 sequencer (Illumina, Inc., USA).

Sequence data were mapped to the reference human genome (hg19) using BWA aligner 0.7.10. Local alignment optimization was performed using GATK 3.2. Germline SNVs were identified using Varscan with default parameters. Germline indels were identified using Varscan and GATK. Pathogenic variants were determined by a clinical molecular geneticist according to the guidelines of the American College of Medical Genetics. ClinVar and Enigma were used during manual curation for final confirmation of the results. The InSIGHT database was used for the pathogenicity classification of the MMR genes.

\section{Prediction of Pathogenicity}

The pathogenicity was predicted for all detected variants using two commonly used tools, SIFT and PolyPhen2.

\section{BRAFVariant Analysis}

In all cases, surgical cancer tissues were used for the BRAF variant analysis. BRAF exon 15 was bidirectionally sequenced using an ABI 3730XL instrument and the BigDye Terminator v. 3.1 cycle sequencing kit (Applied Biosystems, Carlsbad, CA, United States). Three independent experiments were performed to confirm positive samples. DNA from patients was tested using the AmoyDx BRAFvariant 
detection kit (Amoy Diagnostics, Xiamen, China) based on the principles of the amplification-refractory variant system. All results were confirmed according to the criteria suggested by the manufacturer.

\section{Statistical analysis}

Continuous variables were reported as mean \pm standard deviation. Differences in categorical variables and continuous variables between these two groups were analyzed with the Chi square test or Fisher's exact test and with Student's t test, respectively, using the SPSS version 21.0 software (SPSS, Chicago, IL, USA). Twotailed $P$ values less than 0.05 were considered statistically significant.

\section{Results}

\section{Molecular Characteristics}

In the LS group, PVs of $M L H 1$ were identified in 17 (36.2\%) probands, and those of $M S H 2, M S H 6$, and PMS2 were identified in 18 (38.3\%), 10 (21.3\%), and 2 (4.2\%) probands, respectively.

In the LLS group, a total of 52 variants were detected in 44 (54.3\%) individuals, of which eight patients carried multiple variants. Among the 52 variants, 17 were VUS in MMR genes, including 8 in $M L H 1,5$ in $M S H 2,3$ in $M S H 6$, and 1 in PMS2. Of the 17 VUS in the MMR genes, 8 were predicted to be possibly/probably damaging using PolyPhen2, and 4 were predicted to be deleterious using SITF. Other than $M M R$ genes, the most frequently mutated genes were MUYTH, POLE, BRCA2, and GJB2. There were three biallelic missense variants in MUYTH (p.Arg19Ter, p.Gly272G/u, and p.GIn267Ter), two frameshift variants in GJB2 (p.His100fs and p.Leu79Cysfs), and one frameshift variant in RAD50 (p.Glu995fs), which were defined as pathogenic. One case of a missense variant in BUB1B (p.Arg550G/n) was defined as likely pathogenic. Using PolyPhen2, 22 variants were predicted to be possibly/probably damaging, among which most were mutations in the $M L H 1$ and POLE genes. Using SIFT, 20 variants were predicted to be deleterious, among which most were mutations in the POLE, MUTYH, and GJB2 genes. All the variants and prediction of their deleteriousness in the 44 patients from the LLS group are summarized in Table 1. 
Table 1

Variants and prediction of deleteriousness in 44 patients of LLS group.

\begin{tabular}{|c|c|c|c|c|}
\hline Gene & Variants (HGVS) & Clinvar & Polyphen2\# & SIFT* \\
\hline$M L H 1$ & $\begin{array}{l}\text { NC_000003.12:g.37050541T>G } \\
\text { (p.Val720Gly) }\end{array}$ & VUS & $\begin{array}{l}\text { Possibly } \\
\text { damaging } \\
(0.892)\end{array}$ & $\begin{array}{l}\text { Deleterious } \\
(-5.056)\end{array}$ \\
\hline$A P C$ & $\begin{array}{l}\text { NC_000005.10:g.112843926G > T } \\
\text { (p.Ala2778Ser) }\end{array}$ & VUS & $\begin{array}{l}\text { Probably } \\
\text { damaging } \\
(1.000)\end{array}$ & $\begin{array}{l}\text { Neutral } \\
(-0.824)\end{array}$ \\
\hline$M L H 1$ & $\begin{array}{l}\text { NC_000003.11:g.37035002A > G (5 Prime } \\
\text { UTR Variant) }\end{array}$ & VUS & unpredictable & unpredictable \\
\hline$M L H 1$ & $\begin{array}{l}\text { NC_000003.11:g. } 37035004 C>T(5 \text { Prime } \\
\text { UTR Variant) }\end{array}$ & VUS & unpredictable & unpredictable \\
\hline MLH1 & NC_000003.11:g.37035057G > T(p.Val7Phe) & VUS & $\begin{array}{l}\text { Possibly } \\
\text { damaging } \\
(0.485)\end{array}$ & $\begin{array}{l}\text { Neutral } \\
(-1.603)\end{array}$ \\
\hline$M L H 1$ & NC_000003.11:g.37035051G > C(p.Ala5Pro $)$ & VUS & $\begin{array}{l}\text { Possibly } \\
\text { damaging } \\
(0.859)\end{array}$ & $\begin{array}{l}\text { Neutral } \\
(-1.578)\end{array}$ \\
\hline$M L H 1$ & $\begin{array}{l}\text { NC_000003.11:g.37061844A>G } \\
\text { (p.Thr310Ala) }\end{array}$ & VUS & $\begin{array}{l}\text { Probably } \\
\text { damaging } \\
(1.000)\end{array}$ & $\begin{array}{l}\text { Deleterious } \\
(-4.886)\end{array}$ \\
\hline$M L H 1$ & $\begin{array}{l}\text { NC_000003.12:g.37012077A > G } \\
(\text { p.lle219Val) }\end{array}$ & VUS & $\begin{array}{l}\text { Benign } \\
(0.018)\end{array}$ & $\begin{array}{l}\text { Neutral } \\
(-0.460)\end{array}$ \\
\hline$M L H 1$ & $\begin{array}{l}\text { NC_000003.11:g.37092140G >A } \\
\text { (p.Arg687GIn) }\end{array}$ & VUS & $\begin{array}{l}\text { Possibly } \\
\text { damaging } \\
(0.819)\end{array}$ & $\begin{array}{l}\text { Neutral } \\
(-1.040)\end{array}$ \\
\hline $\mathrm{MSH} 2$ & $\begin{array}{l}\text { NC_000001.10:g.236912509G > } \\
\text { T(p.Arg534Leu) }\end{array}$ & VUS & $\begin{array}{l}\text { Probably } \\
\text { damaging } \\
(1.000)\end{array}$ & $\begin{array}{l}\text { Deleterious } \\
(-6.708)\end{array}$ \\
\hline $\mathrm{MSH} 2$ & LRG_218:g.4955G > A (5Prime UTR Variant) & VUS & unpredictable & unpredictable \\
\hline $\mathrm{MSH} 2$ & $\begin{array}{l}\text { NC_000002.11:g.47630246G > A(5 Prime } \\
\text { UTR Variant) }\end{array}$ & VUS & unpredictable & unpredictable \\
\hline $\mathrm{MSH} 2$ & $\begin{array}{l}\text { NC_000002.11:g.47703650G >A } \\
(\text { p.Ser717Asn) }\end{array}$ & VUS & $\begin{array}{l}\text { Benign } \\
(0.263)\end{array}$ & $\begin{array}{l}\text { Neutral } \\
(-2.425)\end{array}$ \\
\hline $\mathrm{MSH} 2$ & $\begin{array}{l}\text { NC_.000002.11:g.47703539G > } \\
\text { C(p.Arg680Pro })\end{array}$ & VUS & $\begin{array}{l}\text { Probably } \\
\text { damaging } \\
(1.000)\end{array}$ & $\begin{array}{l}\text { Deleterious } \\
(-6.534)\end{array}$ \\
\hline MSH6 & $\begin{array}{l}\text { NC_000002.12:g.47783304C> } \\
T(\text { p.Ser24Leu })\end{array}$ & VUS & $\begin{array}{l}\text { Benign } \\
(0.007)\end{array}$ & $\begin{array}{l}\text { Neutral } \\
(-0.535)\end{array}$ \\
\hline
\end{tabular}

\# These data are presented as prediction (predicted score); *these data are presented as prediction (PROVEAN score). 


\begin{tabular}{|c|c|c|c|c|}
\hline Gene & Variants (HGVS) & Clinvar & Polyphen2 ${ }^{\#}$ & SIFT* \\
\hline MSH6 & $\begin{array}{l}\text { NC_000002.11:g.48033801_48033825del } \\
\text { (Splice Donor Variant) }\end{array}$ & VUS & unpredictable & unpredictable \\
\hline RAD50 & $\begin{array}{l}\text { NC_000005.10:g.132609343_132609346del } \\
\text { (p.Glu995fs) }\end{array}$ & Pathogenic & unpredictable & $\begin{array}{l}\text { Deleterious } \\
(-6.228)\end{array}$ \\
\hline MSH6 & $\begin{array}{l}\text { NC_000002.12:g.47795968C> } \\
\text { T(p.Arg178Cys) }\end{array}$ & VUS & $\begin{array}{l}\text { Probably } \\
\text { damaging } \\
(0.974)\end{array}$ & $\begin{array}{l}\text { Neutral } \\
(-1.577)\end{array}$ \\
\hline NSD1 & $\begin{array}{l}\text { NC_000005.10:g.177211614G >A } \\
(\text { (p.Arg1072G/n) }\end{array}$ & VUS & $\begin{array}{l}\text { Benign } \\
(0.009)\end{array}$ & $\begin{array}{l}\text { Deleterious } \\
(0.041)\end{array}$ \\
\hline PMS2 & $\begin{array}{l}\text { NC_000007.14:g.5987462G >A } \\
\text { (p.His435Tyr) }\end{array}$ & VUS & $\begin{array}{l}\text { Benign } \\
(0.017)\end{array}$ & $\begin{array}{l}\text { Neutral } \\
(-0.795)\end{array}$ \\
\hline MUTYH & $\begin{array}{l}\text { NC_000001.10:g.45800123G >A } \\
\text { (p.Arg19Ter) }\end{array}$ & Pathogenic & unpredictable & $\begin{array}{l}\text { Deleterious } \\
(-2.625)\end{array}$ \\
\hline MUTYH & $\begin{array}{l}N C \_000001.10: g .45797914 C>T \\
(p . G l y 272 G l u)\end{array}$ & Pathogenic & $\begin{array}{l}\text { Probably } \\
\text { damaging } \\
(1.000)\end{array}$ & $\begin{array}{l}\text { Deleterious } \\
(-7.553)\end{array}$ \\
\hline MUTYH & $\begin{array}{l}\text { NC_000001.10:g.45797972G > } \\
\text { A(p.GIn267Ter) }\end{array}$ & Pathogenic & unpredictable & $\begin{array}{l}\text { Deleterious } \\
(-9.745)\end{array}$ \\
\hline MUTYH & $\begin{array}{l}\text { NC_000001.11:g.45334474C> } \\
\text { T(p.Pro18Leu })\end{array}$ & VUS & $\begin{array}{l}\text { Benign } \\
(0.006)\end{array}$ & $\begin{array}{l}\text { Neutral } \\
(-1.436)\end{array}$ \\
\hline MUTYH & $\begin{array}{l}\text { NC_000001.10:g.45797760T > C(5 Prime } \\
\text { UTR Variant) }\end{array}$ & VUS & unpredictable & unpredictable \\
\hline TP53 & NC_000017.10:g.7579705C>T (p.Val31lle) & VUS & $\begin{array}{l}\text { Benign } \\
(0.001)\end{array}$ & $\begin{array}{l}\text { Neutral } \\
(-0.142)\end{array}$ \\
\hline GJB2 & $\begin{array}{l}\text { NC_000013.10:g.20763421_20763422del } \\
\text { (p.His100fs) }\end{array}$ & Pathogenic & unpredictable & $\begin{array}{l}\text { Neutral } \\
(-2.065)\end{array}$ \\
\hline GJB2 & NC_000013.10:g.20763488del (p.Leu79fs) & Pathogenic & unpredictable & $\begin{array}{l}\text { Deleterious } \\
(-13.990)\end{array}$ \\
\hline GJB2 & NC_000013.10.20763486G>-(p.Leu79Cysfs) & Pathogenic & unpredictable & $\begin{array}{l}\text { Deleterious } \\
(-13.990)\end{array}$ \\
\hline GJB2 & $\begin{array}{l}\text { NC_000013.10:g.20763150A > G } \\
\text { (p.Phe191Leu) }\end{array}$ & VUS & $\begin{array}{l}\text { Probably } \\
\text { damaging } \\
(1.000)\end{array}$ & $\begin{array}{l}\text { Deleterious } \\
(-5.719)\end{array}$ \\
\hline ATM & $\begin{array}{l}\text { NC_000011.10:g.108250816C> } \\
\text { T(p.Arg451Cys) }\end{array}$ & VUS & $\begin{array}{l}\text { Probably } \\
\text { damaging } \\
(1.000)\end{array}$ & $\begin{array}{l}\text { Neutral - } \\
2.171\end{array}$ \\
\hline
\end{tabular}

\# These data are presented as prediction (predicted score); *these data are presented as prediction (PROVEAN score). 


\begin{tabular}{|c|c|c|c|c|}
\hline Gene & Variants (HGVS) & Clinvar & Polyphen2 ${ }^{\#}$ & SIFT* \\
\hline POLE & $\begin{array}{l}\text { NC_000012.11:g.133215747C> } \\
\text { T(p.Arg1839His) }\end{array}$ & VUS & $\begin{array}{l}\text { Probably } \\
\text { damaging } \\
(0.993)\end{array}$ & $\begin{array}{l}\text { Deleterious } \\
(-4.652)\end{array}$ \\
\hline POLE & $\begin{array}{l}\text { NC_000012.11:g.133225520G > } \\
\text { A(p.Arg1382Cys) }\end{array}$ & VUS & $\begin{array}{l}\text { Probably } \\
\text { damaging } \\
(0.994)\end{array}$ & $\begin{array}{l}\text { Deleterious } \\
(-5.698)\end{array}$ \\
\hline POLE & $\begin{array}{l}\text { NC_000012.11:g.133201290C> } \\
T(\text { p.Gly2285Asp) }\end{array}$ & VUS & $\begin{array}{l}\text { Benign } \\
(0.000)\end{array}$ & $\begin{array}{l}\text { Neutral } \\
(-0.437)\end{array}$ \\
\hline POLE & $\begin{array}{l}\text { NC_000012.11:g.133250198G > } \\
\text { A(p.Pro441Leu) }\end{array}$ & VUS & $\begin{array}{l}\text { Probably } \\
\text { damaging } \\
(1.000)\end{array}$ & $\begin{array}{l}\text { Deleterious } \\
(-8.776)\end{array}$ \\
\hline POLE & $\begin{array}{l}\text { NC_000003.11:g.37090494C> } \\
\text { G(p.Pro697Ala) }\end{array}$ & VUS & $\begin{array}{l}\text { Benign } \\
(0.000)\end{array}$ & $\begin{array}{l}\text { Deleterious } \\
(-3.425)\end{array}$ \\
\hline POLE & $\begin{array}{l}\text { NC_000012.12:g.132676107T > } \\
C(\text { p.Asn336Ser) }\end{array}$ & VUS & $\begin{array}{l}\text { Probably } \\
\text { damaging } \\
(1.000)\end{array}$ & $\begin{array}{l}\text { Deleterious } \\
(-4.472)\end{array}$ \\
\hline POLD1 & $\begin{array}{l}\text { NC_000023.10:g.152034432G }>A \\
\text { (p.Gly205Ser) }\end{array}$ & VUS & $\begin{array}{l}\text { Benign } \\
(0.150)\end{array}$ & $\begin{array}{l}\text { Neutral } \\
(-0.030)\end{array}$ \\
\hline$B R C A 1$ & $\begin{array}{l}\text { NC_000017.11:g.43106514G > } \\
\text { A(p.Leu52Phe) }\end{array}$ & VUS & $\begin{array}{l}\text { Probably } \\
\text { damaging } \\
(1.000)\end{array}$ & $\begin{array}{l}\text { Neutral } \\
(-0.587)\end{array}$ \\
\hline BRCA2 & $\begin{array}{l}\text { NC_000013.10:g.32913723G > } \\
\text { T(p.Ser1744lle) }\end{array}$ & VUS & $\begin{array}{l}\text { Benign } \\
(0.048)\end{array}$ & $\begin{array}{l}\text { Neutral } \\
(-2.182)\end{array}$ \\
\hline BRCA2 & $\begin{array}{l}\text { NC_000013.10:g.32906967G > } \\
\text { A(p.Ser451Asn) }\end{array}$ & VUS & $\begin{array}{l}\text { Benign } \\
(0.009)\end{array}$ & $\begin{array}{l}\text { Neutral } \\
(-0.431)\end{array}$ \\
\hline ATR & $\begin{array}{l}\text { NC_000003.11:g. } 142172064 G> \\
\text { C(p.Thr2556Ser) }\end{array}$ & VUS & $\begin{array}{l}\text { Possibly } \\
\text { damaging } \\
(0.830)\end{array}$ & $\begin{array}{l}\text { Neutral } \\
(-1.301)\end{array}$ \\
\hline EPCAM & $\begin{array}{l}\text { NC_000002.11:g.47600631G >A } \\
\text { (p.Val36lle) }\end{array}$ & VUS & $\begin{array}{l}\text { Benign } \\
(0.002)\end{array}$ & $\begin{array}{l}\text { Neutral } \\
(-0.350)\end{array}$ \\
\hline MSH3 & $\begin{array}{l}\text { NC_000005.10:g.80813659T > } \\
\text { G(p.Leu911Val) }\end{array}$ & VUS & $\begin{array}{l}\text { Benign } \\
(0.174)\end{array}$ & $\begin{array}{l}\text { Deleterious } \\
(-2.721)\end{array}$ \\
\hline PMS1 & $\begin{array}{l}\text { NC_000002.11:g.190649220G > A(5 Prime } \\
\text { UTR Variant) }\end{array}$ & VUS & unpredictable & unpredictable \\
\hline APC & $\begin{array}{l}\text { NC_000005.10:g.112843926G > } \\
\text { T(p.Ala2778Ser) }\end{array}$ & VUS & $\begin{array}{l}\text { Probably } \\
\text { damaging } \\
(1.000)\end{array}$ & $\begin{array}{l}\text { Neutral } \\
(-0.824)\end{array}$ \\
\hline SMAD4 & $\begin{array}{l}\text { NC_000018.10:g.51059908A > G } \\
\text { (p.Asn316Ser) }\end{array}$ & VUS & $\begin{array}{l}\text { Benign } \\
(0.002)\end{array}$ & $\begin{array}{l}\text { Neutral } \\
(-0.829)\end{array}$ \\
\hline
\end{tabular}




\begin{tabular}{|c|c|c|c|c|}
\hline Gene & Variants (HGVS) & Clinvar & Polyphen2\# & SIFT* \\
\hline BUB1B & $\begin{array}{l}\text { NC_000015.10:g.40202609G > } \\
\text { A(p.Arg550Gln) }\end{array}$ & $\begin{array}{l}\text { Likely } \\
\text { benign }\end{array}$ & $\begin{array}{l}\text { Benign } \\
(0.001)\end{array}$ & $\begin{array}{l}\text { Neutral } \\
(0.332)\end{array}$ \\
\hline $\mathrm{CDH} 1$ & $\begin{array}{l}\text { NC_000016.10:g.68811716G >A } \\
\text { (p.Ala289Thr) }\end{array}$ & VUS & $\begin{array}{l}\text { Probably } \\
\text { damaging } \\
(0.932)\end{array}$ & $\begin{array}{l}\text { Neutral } \\
(-2.459\end{array}$ \\
\hline $\mathrm{CDH} 1$ & $\begin{array}{l}\text { NC_000016.9:g.68856080C > } \\
\text { G(p.Leu630Val) }\end{array}$ & VUS & $\begin{array}{l}\text { Probably } \\
\text { damaging } \\
(0.998)\end{array}$ & $\begin{array}{l}\text { Deleterious } \\
(-2.726)\end{array}$ \\
\hline CHEK2 & $\begin{array}{l}\text { NC_000022.10:g.29083956G >A } \\
\text { (p.Arg521Trp) }\end{array}$ & VUS & $\begin{array}{l}\text { Probably } \\
\text { damaging } \\
(1.000)\end{array}$ & $\begin{array}{l}\text { Deleterious } \\
(-4.126)\end{array}$ \\
\hline DICER1 & $\begin{array}{l}\text { NC_000014.8:g.95590896T > } \\
\text { G(p.Glu338Ala) }\end{array}$ & VUS & $\begin{array}{l}\text { Benign } \\
(0.310)\end{array}$ & $\begin{array}{l}\text { Neutral } \\
(-0.642)\end{array}$ \\
\hline
\end{tabular}

The distribution of $M M R$ deficiencies in the two groups was compared, and the results are summarized in Table 2. A total of $19.1 \%(9 / 46)$ of the patients in the LS group manifested deficiency in MSH6 by immunohistochemistry, which was significantly higher than that $(7.4 \%, 6 / 81)$ in the LLS group $\left(\chi^{2}=3.963, P\right.$ $=0.046)$. No significant differences were observed in case of other $M M R$ deficiencies. 
Table 2

Distribution of $M M R$ deficiency in the two groups.

\begin{tabular}{|c|c|c|c|c|}
\hline MMR deficiency & $\begin{array}{l}\text { LS group } \\
(\mathrm{N}=47)\end{array}$ & $\begin{array}{l}\text { LLS group } \\
(\mathrm{N}=81)\end{array}$ & $\chi^{2}$ value & $\mathrm{p}$ value \\
\hline MLH1/PMS2 & & & 0.482 & 0.487 \\
\hline Presence & $14(29.8 \%)$ & $29(35.8 \%)$ & & \\
\hline MSH2/MSH6 & & & 0.182 & 0.670 \\
\hline Presence & $12(25.5 \%)$ & $18(22.2 \%)$ & & \\
\hline Isolated $M L H 1$ & & & 0.504 & 0.478 \\
\hline Presence & $2(4.3 \%)$ & $6(7.4 \%)$ & & \\
\hline Isolated $M S H 2$ & & & 0.019 & 0.891 \\
\hline Presence & $5(10.6 \%)$ & $8(9.9 \%)$ & & \\
\hline Isolated MSH6 & & & 3.963 & 0.046 \\
\hline Presence & $9(19.1 \%)$ & $6(7.4 \%)$ & & \\
\hline Isolated PMS2 & & & 2.044 & 0.153 \\
\hline Presence & $3(6.4 \%)$ & $12(14.8 \%)$ & & \\
\hline Other & & & 0.313 & 0.576 \\
\hline Presence & $2(4.3 \%)$ & $2(2.5 \%)$ & & \\
\hline
\end{tabular}

\section{Demographic And Clinical Characteristics}

The demographic and clinical characteristics of the 128 enrolled patients were compared between the LS and LLS groups and are summarized in Table 3. There were significant differences in the proportion of patients with the earliest onset age of CRC and in the primary CRC location between the two groups. In the LS group, $74.5 \%$ (35/47) of the patients were characterized by early-onset ( $<50$ years old) CRC, which was significantly higher than the proportion $(53.1 \%, 43 / 81)$ found in the LLS group $\left(\chi^{2}=5.712, P=0.017\right)$. In the LLS group, $25.9 \%$ (21/81) of the patients developed primary CRC in the rectum, which was remarkably higher than the proportion $(10.6 \%, 5 / 47)$ found in the $L S$ group $\left(X^{2}=2.358, P=0.046\right)$. 
Table 3

Demographic and clinical characteristics of 128 patients with colorectal cancer in the two groups.

\begin{tabular}{|c|c|c|c|c|}
\hline Characteristic & $\begin{array}{l}\text { LS group } \\
(N=47)\end{array}$ & $\begin{array}{l}\text { LLS group } \\
(\mathrm{N}=81)\end{array}$ & $\begin{array}{l}\chi^{2} / t \\
\text { value }\end{array}$ & $\begin{array}{l}P \\
\text { value }\end{array}$ \\
\hline Gender & & & 0.985 & 0.321 \\
\hline Male & $26(55.3 \%)$ & $52(64.2 \%)$ & & \\
\hline Female & $21(44.7 \%)$ & $29(35.8 \%)$ & & \\
\hline Age(years) ${ }^{a}$ & $\begin{array}{l}44.36 \pm \\
11.26\end{array}$ & $\begin{array}{l}48.12 \pm \\
13.09\end{array}$ & -1.715 & 0.089 \\
\hline$<50$ & $35(74.5 \%)$ & $43(53.1 \%)$ & 5.712 & 0.017 \\
\hline$\geq 50$ & $12(2.5 .5 \%)$ & $38(46.9 \%)$ & & \\
\hline Diagnostic criteria & & & 4.297 & 0.117 \\
\hline Amsterdam I & $11(23.4 \%)$ & $9(11.1 \%)$ & & \\
\hline Amsterdam II & $23(43.9 \%)$ & $39(48.1 \%)$ & & \\
\hline Bethesda & $13(27.7 \%)$ & $33(40.8)$ & & \\
\hline CEA (ng/ml) & & & 0.145 & 0.714 \\
\hline$<5.2$ & $7(14.9 \%)$ & $15(18.5 \%)$ & & \\
\hline$\geq 5.2$ & $40(85.1 \%)$ & $66(81.5 \%)$ & & \\
\hline Location of colorectal cancer & & & 7.994 & 0.046 \\
\hline Right colon & $18(38.3 \%)$ & $32(39.5 \%)$ & & \\
\hline Left colon & $20(42.6 \%)$ & $19(23.5 \%)$ & & \\
\hline Rectal & $5(10.6 \%)$ & $21(25.9 \%)$ & & \\
\hline Multiple & $4(8.5 \%)$ & $9(11.1 \%)$ & & \\
\hline Multiple tumors & & & 2.358 & 0.125 \\
\hline Occurrence & $12(25.5 \%)$ & $12(14.8 \%)$ & & \\
\hline Absence & $35(74.5 \%)$ & $69(85.2 \%)$ & & \\
\hline Tumor size ${ }^{\mathrm{a}}(\mathrm{cm})$ & $5.17 \pm 2.61$ & $5.11 \pm 2.49$ & 0.127 & 0.899 \\
\hline Pathological classification & & & 5.156 & 0.076 \\
\hline
\end{tabular}

a These data are presented as mean \pm standard deviation; other values are presented as number of patients followed by percentage in parentheses.

LS: Lynch syndrome, LLS: Lynch-like syndrome. 


\begin{tabular}{|c|c|c|c|c|}
\hline Characteristic & $\begin{array}{l}\text { LS group } \\
(N=47)\end{array}$ & $\begin{array}{l}\text { LLS group } \\
(\mathrm{N}=81)\end{array}$ & $\begin{array}{l}\chi^{2} / t \\
\text { value }\end{array}$ & $\begin{array}{l}P \\
\text { value }\end{array}$ \\
\hline Adenocarcinoma & $34(72.3 \%)$ & $66(81.5 \%)$ & & \\
\hline $\begin{array}{l}\text { Adenocarcinoma with partial mucinous } \\
\text { adenocarcinoma }\end{array}$ & $5(10.7 \%)$ & $11(13.6 \%)$ & & \\
\hline Mucinous adenocarcinoma & $8(17.0 \%)$ & $4(4.9 \%)$ & & \\
\hline Differentiation & & & 0.365 & 0.833 \\
\hline Well differentiated & $1(2.1 \%)$ & $3(3.7 \%)$ & & \\
\hline Moderately differentiated & $28(59.6 \%)$ & $45(55.6 \%)$ & & \\
\hline Poorly differentiated & $18(38.3 \%)$ & $33(40.7 \%)$ & & \\
\hline Cancerous node & & & 1.780 & 0.182 \\
\hline Occurrence & $2(4.3 \%)$ & $9(11.1 \%)$ & & \\
\hline Absence & $45(95.7 \%)$ & $72(88.9 \%)$ & & \\
\hline Vascular invasion & & & 0.045 & 0.832 \\
\hline Occurrence & $8(17.0 \%)$ & $15(18.5 \%)$ & & \\
\hline Absence & $39(83.0 \%)$ & $66(81.5 \%)$ & & \\
\hline Perineural invasion & & & 0.079 & 0.779 \\
\hline Occurrence & $6(12.8 \%)$ & $9(11.1 \%)$ & & \\
\hline Absence & $41(87.2 \%)$ & $72(88.9 \%)$ & & \\
\hline T stage & & & 0.804 & 0.669 \\
\hline $\mathrm{T} 1$ & $7(14.9 \%)$ & $8(9.9 \%)$ & & \\
\hline T2 & $8(17.0 \%)$ & $13(16.0 \%)$ & & \\
\hline T3 & $32(68.1 \%)$ & $60(74.1 \%)$ & & \\
\hline $\mathrm{N}$ stage & & & 0.911 & 0.634 \\
\hline NO & $34(72.3 \%)$ & $53(65.4 \%)$ & & \\
\hline N1 & $9(19.1 \%)$ & $17(21.0 \%)$ & & \\
\hline $\mathrm{N} 2$ & $4(8.6 \%)$ & $11(13.6 \%)$ & & \\
\hline
\end{tabular}

\footnotetext{
a These data are presented as mean \pm standard deviation; other values are presented as number of patients followed by percentage in parentheses.
}

LS: Lynch syndrome, LLS: Lynch-like syndrome. 


\begin{tabular}{|c|c|c|c|c|}
\hline Characteristic & $\begin{array}{l}\text { LS group } \\
(\mathrm{N}=47)\end{array}$ & $\begin{array}{l}\text { LLS group } \\
(\mathrm{N}=\mathbf{8 1})\end{array}$ & $\begin{array}{l}\chi^{2} / t \\
\text { value }\end{array}$ & $\begin{array}{l}P \\
\text { value }\end{array}$ \\
\hline Metastasis & & & 0.313 & 0.576 \\
\hline Occurrence & $2(4.3 \%)$ & $3(3.7 \%)$ & & \\
\hline Absence & $45(95.7 \%)$ & 78(96.3\%) & & \\
\hline TNM stage & & & 1.152 & 0.764 \\
\hline I & $13(27.7 \%)$ & 16(19.8\%) & & \\
\hline ॥ & 17(36.2\%) & $32(39.5 \%)$ & & \\
\hline III & 15(31.8\%) & $30(37.0 \%)$ & & \\
\hline IV & $2(4.3 \%)$ & $3(3.7 \%)$ & & \\
\hline \multicolumn{5}{|c|}{$\begin{array}{l}\text { a These data are presented as mean } \pm \text { standard deviation; other values are presented as number of } \\
\text { patients followed by percentage in parentheses. }\end{array}$} \\
\hline
\end{tabular}

\section{Pathological Characteristics}

Comparison of the pathological results showed no significant differences in the pathological TNM stage $\left(\chi^{2}\right.$ $=1.152, P=0.764)$ and differentiation of the $C R C$ tumors $\left(X^{2}=0.365, P=0.833\right)$ between the two groups. The proportion of patients with mucinous CRC was $17.0 \%$ (8/47) in the LS group, which was higher than that $(4.9 \%, 4 / 81)$ in the LLS group, whereas the proportions of patients with adenocarcinoma and partial mucinous CRC were similar between the two groups. Thus, no significant differences were observed in pathological classification $\left(X^{2}=5.516, P=0.076\right)$. The pathological characteristics of the CRC tumors in the two groups are summarized in Table 3 .

\section{Primary And Metachronous Crc In Probands}

During the follow-up period, $34.0 \%$ (16/47) of the patients in the LS group and $38.3 \%(31 / 81)$ in the LLS group developed metachronous $\mathrm{CRC}$, with no significant difference observed between the groups $\left(\chi^{2}=\right.$ $0.229, P=0.632$ ). The period between the occurrence of primary and metachronous CRC was $28.78 \pm 29.14$ months in the LS group and $38.58 \pm 24.89$ months in the LLS group, with no significant difference being observed between the groups $(t=-1.033, P=0.108)$.

The mean age of cancer onset was $43.40 \pm 11.17$ years in the LS group and significantly higher ( $47.56 \pm$ 12.99 years $)$ in the LLS group $(t=-2.008, P=0.049)$. The locations of the metachronous $C R C$ tumors were similar to those of primary CRC. In the LLS group, 38.3\% (31/81) of the patients developed rectal cancer, 
which was markedly higher than the proportion $(17.0 \%, 8 / 47)$ found in the LS group $\left(\chi^{2}=6.340, P=0.012\right)$. The tumor histories in the probands from the two groups are summarized in Table 4.

Table 4

Comparison of patients' tumor histories between LS group and LLS group.

\begin{tabular}{|c|c|c|c|c|}
\hline Characteristic & $\begin{array}{l}\text { LS group } \\
(\mathrm{N}=47)\end{array}$ & $\begin{array}{l}\text { LLS group } \\
(\mathrm{N}=81)\end{array}$ & $\begin{array}{l}\chi^{2} / t \\
\text { value }\end{array}$ & $\begin{array}{l}\mathrm{p} \\
\text { value }\end{array}$ \\
\hline Earliest onset age of CRC (years) ${ }^{a}$ & $\begin{array}{l}44.36 \pm \\
11.26\end{array}$ & $\begin{array}{l}48.12 \pm \\
13.09\end{array}$ & -1.175 & 0.089 \\
\hline Total number of $C R C s^{a}$ & $1.55 \pm 0.75$ & $1.51 \pm 0.59$ & 0.392 & 0.696 \\
\hline Metachronous CRC & & & 0.229 & 0.632 \\
\hline Occurrence & $16(34.0 \%)$ & $31(38.3 \%)$ & & \\
\hline Right colon cancer & & & 0.001 & 0.972 \\
\hline Occurrence & $28(59.6 \%)$ & $48(59.3 \%)$ & & \\
\hline Left colon cancer & & & 2.505 & 0.113 \\
\hline Occurrence & $30(63.8 \%)$ & $40(49.4 \%)$ & & \\
\hline Rectal cancer & & & 6.340 & 0.012 \\
\hline Occurrence & $8(17.0 \%)$ & $31(38.3 \%)$ & & \\
\hline Synchronous or metachronous CRC & & & 0.118 & 0.874 \\
\hline Occurrence & $20(42.6 \%)$ & $37(45.7 \%)$ & & \\
\hline $\begin{array}{l}\text { Earliest onset age of extra-colorectal cancer } \\
\left(\text { years) }{ }^{a \star}\right.\end{array}$ & $\begin{array}{l}48.45 \pm \\
12.68\end{array}$ & $\begin{array}{l}49.79 \pm \\
10.28\end{array}$ & -0.345 & 0.732 \\
\hline $\begin{array}{l}\text { Synchronous or metachronous extra-colorectal } \\
\text { cancer }\end{array}$ & & & 2.128 & 0.145 \\
\hline Occurrence & $11(23.4 \%)$ & $29(35.8 \%)$ & & \\
\hline Earliest onset age of cancer (years) ${ }^{a}$ & $\begin{array}{l}43.40 \pm \\
11.17\end{array}$ & $\begin{array}{l}47.56 \pm \\
12.99\end{array}$ & -2.008 & 0.049 \\
\hline Total number of cancers ${ }^{a}$ & $1.89 \pm 1.03$ & $1.96 \pm 0.94$ & -0.389 & 0.698 \\
\hline
\end{tabular}

\section{Extra-crc In Probands}


In the LS group, 11 patients developed 15 cases of primary extra-CRC, including 5 cases of endometrial cancer, 5 cases of gastric cancer, 2 cases of small intestinal cancer, and 1 case each of ovarian, breast, and cutaneous cancer. In the LLS group, 29 patients developed 29 cases of extra-CRCs, including 8 cases of gastric cancer, 6 cases of endometrial cancer, 4 cases each of small intestinal and breast cancer, 2 cases each of prostate and ovarian cancer, and 1 case each of ureteral carcinoma, renal cancer, and pancreatic cancer. The proportions of synchronous or metachronous extra-CRC were $23.4 \%(11 / 47)$ in the LS group and $35.8 \%(29 / 81)$ in the LLS group, with no significant difference observed between the groups $\left(X^{2}=2.128\right.$, $\mathrm{P}=0.145)$.

\section{Family Pedigrees}

A total of 142 first- and second-degree relatives who developed LS-associated cancer in the LS families and 210 of those in the LLS families were enrolled in the pedigree analysis.

In the LS families, the mean number of patients who developed CRC was $3.26 \pm 2.08$, which was significantly higher than that $(2.42 \pm 1.65)$ in the LLS families $(t=2.506, P=0.013)$. The mean earliest age of CRC onset was $37.53 \pm 8.63$ years in the LS families, which was significantly lower than that (44.51 \pm 13.64 years) in the LLS families ( $t=-3.156, P=0.002)$. In terms of the tumor distribution, left colon cancer was observed in $91.5 \%(43 / 47)$ of the LS families, which was significantly more frequent than that $(70.4 \%$, $57 / 81)$ in the LLS families $\left(X^{2}=7.762, P=0.005\right)$.

In addition to CRC, the mean number of patients who developed extra-CRC was $1.59 \pm 1.38$ in the LLS families, which was significantly higher than that $(1.09 \pm 1.37)$ in the LS families $(t=-2.017, P=0.045)$. The pedigrees of the LS and LLS families were compared, and the results are summarized in Table 5. 
Table 5

Comparison of pedigrees between the LS group and LLS group.

\begin{tabular}{|c|c|c|c|c|}
\hline Variable & $\begin{array}{l}\text { LS group } \\
(\mathrm{N}=47)\end{array}$ & $\begin{array}{l}\text { LLS group } \\
(N=46)\end{array}$ & $\begin{array}{l}t / \chi^{2} \\
\text { value }\end{array}$ & $\begin{array}{l}\mathrm{p} \\
\text { value }\end{array}$ \\
\hline Patients with cancer (cases) ${ }^{a}$ & $4.02 \pm 2.48$ & $3.59 \pm 1.99$ & 1.073 & 0.285 \\
\hline Male patients(cases) ${ }^{a}$ & $2.28 \pm 1.72$ & $2.04 \pm 1.63$ & 0.786 & 0.433 \\
\hline Female patients (cases) ${ }^{a}$ & $1.74 \pm 1.42$ & $1.54 \pm 1.32$ & 0.808 & 0.421 \\
\hline First degree relatives (cases) ${ }^{\mathrm{a}}$ & $1.98 \pm 1.69$ & $1.79 \pm 1.58$ & 0.635 & 0.526 \\
\hline Second degree relatives (cases) ${ }^{a}$ & $1.04 \pm 1.55$ & $0.80 \pm 1.23$ & 0.968 & 0.335 \\
\hline Cases of cancer ${ }^{\mathrm{a}}$ & $5.13 \pm 3.10$ & $4.83 \pm 2.84$ & 0.558 & 0.578 \\
\hline Patients with CRC (cases) ${ }^{a}$ & $3.26 \pm 2.08$ & $2.42 \pm 1.65$ & 2.506 & 0.013 \\
\hline Cases of $\mathrm{CRC}^{\mathrm{a}}$ & $3.91 \pm 2.54$ & $3.12 \pm 2.40$ & 2.109 & 0.047 \\
\hline Patients with right colon cancer (cases) ${ }^{a}$ & $1.45 \pm 1.02$ & $1.27 \pm 1.13$ & 0.877 & 0.382 \\
\hline Cases of right colon cancer ${ }^{a}$ & $1.49 \pm 1.06$ & $1.32 \pm 1.31$ & 0.749 & 0.455 \\
\hline Right colon cancer & & & 1.356 & 0.244 \\
\hline Occurrence & $38(80.9 \%)$ & $58(71.6 \%)$ & & \\
\hline Patients with left colon cancer (cases) ${ }^{a}$ & $1.72 \pm 1.19$ & $0.98 \pm 0.87$ & 3.764 & $\begin{array}{l}<.001 \\
0.001\end{array}$ \\
\hline Cases of left colon cancer ${ }^{a}$ & $1.94 \pm 1.54$ & $1.04 \pm 1.01$ & 3.588 & 0.001 \\
\hline Left colon cancer & & & 7.762 & 0.005 \\
\hline Occurrence & $43(91.5 \%)$ & $57(70.4 \%)$ & & \\
\hline Patients with rectal cancer (cases) ${ }^{a}$ & $0.49 \pm 0.69$ & $0.77 \pm 0.99$ & -1.688 & 0.094 \\
\hline Cases of rectal cancer ${ }^{a}$ & $0.49 \pm 0.69$ & $0.77 \pm 0.99$ & -1.688 & 0.094 \\
\hline Rectal cancer & & & 2.608 & 0.106 \\
\hline Occurrence & $18(38.3 \%)$ & $43(53.1 \%)$ & & \\
\hline Patients with extra-colorectal cancer (cases) ${ }^{a}$ & $1.09 \pm 1.37$ & $1.59 \pm 1.38$ & -2.017 & 0.046 \\
\hline
\end{tabular}

a These data are presented as mean \pm standard deviation; other values are presented as number of patients followed by percentage in parentheses. * These data are limited to families that developed extra-colorectal cancer.

LS: Lynch syndrome, LLS: Lynch-like syndrome, CRC: colorectal cancer. 


\begin{tabular}{|c|c|c|c|c|}
\hline Variable & $\begin{array}{l}\text { LS group } \\
(\mathrm{N}=47)\end{array}$ & $\begin{array}{l}\text { LLS group } \\
(\mathrm{N}=46)\end{array}$ & $\begin{array}{l}\mathrm{t} / \mathrm{x}^{2} \\
\text { value }\end{array}$ & $\begin{array}{l}\mathrm{p} \\
\text { value }\end{array}$ \\
\hline Cases of extra-colorectal cancers ${ }^{a}$ & $1.21 \pm 1.49$ & $1.70 \pm 1.52$ & -2.005 & 0.045 \\
\hline Extra-colorectal cancer & & & 1.140 & 0.286 \\
\hline Occurrence & $30(63.8 \%)$ & $59(72.8 \%)$ & & \\
\hline Synchronous or metachronous CRC & & & 0.060 & 0.807 \\
\hline Occurrence & $21(44.7 \%)$ & $38(46.9 \%)$ & & \\
\hline $\begin{array}{l}\text { Synchronous or metachronous extra-colorectal } \\
\text { cancer }\end{array}$ & & & 1.948 & 0.163 \\
\hline Occurrence & $15(31.9 \%)$ & $36(44.4 \%)$ & & \\
\hline Earliest onset age of cancer (years) ${ }^{a}$ & $36.66 \pm 8.75$ & $\begin{array}{l}41.60 \pm \\
11.91\end{array}$ & -2.690 & 0.008 \\
\hline Earliest onset age of CRC (years) ${ }^{a}$ & $37.53 \pm 8.63$ & $\begin{array}{l}44.51 \pm \\
13.64\end{array}$ & -3.156 & 0.002 \\
\hline $\begin{array}{l}\text { Earliest onset age of extra-colorectal cancer } \\
\text { (years)* }\end{array}$ & $\begin{array}{l}45.00 \pm \\
10.27\end{array}$ & $\begin{array}{l}47.97 \pm \\
10.09\end{array}$ & -1.303 & 0.196 \\
\hline \multicolumn{5}{|c|}{$\begin{array}{l}\text { a These data are presented as mean } \pm \text { standard deviation; other values are presented as number of } \\
\text { patients followed by percentage in parentheses. }{ }^{*} \text { These data are limited to families that developed } \\
\text { extra-colorectal cancer. }\end{array}$} \\
\hline LS: Lynch syndrome, LLS: Lynch-like syndrome & colorectal c & & & \\
\hline
\end{tabular}

Analysis of the sex distribution showed that the mean number of the male patients in the LLS families was $2.04 \pm 1.63$, which was significantly higher than that $(1.54 \pm 1.32)$ of the female patients $(t=2.116, P=$ 0.036 ). In the LS families, the mean numbers of the male and female patients were $2.28 \pm 1.72$ and $1.74 \pm$ 1.42 , respectively, with no significant difference being observed $(t=1.637, P=0.105)$.

\section{Discussion}

With respect to oncologic outcomes, MMR-deficient CRC is associated with a better prognosis and therapeutic responses because the $M M R$ pathway is involved in triggering cell death after chemotherapyinduced DNA damage [14]. The prognosis in patients with MMR-deficient CRC tends to be better, with regard to stage-for-stage comparison, than in those with MMR-proficient cancer [14]. Patients with early-stage $M M R$-deficient CRC do not appear to benefit from adjuvant 5-Fluorouracil monotherapy [15]; however, in some patients with metastatic MMR-deficient CRC, treatment with immune checkpoint inhibitors has been associated with an excellent response [16].

However, a considerable number of MMR-deficient CRC tumors have an unknown etiology, other than confirmed LS and methylation of MLH1. In our study, a high proportion of patients with MMR-deficient CRC 
were diagnosed as having LLS, which was consistent with the data of a previous study [9]. Therefore, multigene panel testing should be recommended for all MMR-deficient patients to distinguish LS and LLS.

While management of LS has been well described, the inability to define the molecular basis of the LLS entity not only hampers the appropriate clinical management of probands, but also the cancer screening recommendations for affected families. Comparison of clinical and molecular characteristics of patients with LLS and features of their CRC tumors with those of confirmed patients with LS can contribute to the development of appropriate management recommendations for patients with LLS and their affected family members.

The genetic causes of LLS are still unknown, although advanced NGS approaches have facilitated the discovery of novel genetic events that may allow the definition of clinical and molecular phenotypes of LLS. In our study, variants were unidentified in nearly half of the LLS cohort. Current techniques of analysis may be missing complex or cryptic variants in $M M R$ genes, and some deep intronic variants may be overlooked $[17,18]$. Furthermore, there may be some unidentified variants in the regulatory regions of $M M R$ genes, which are hardly screened [19]. Thus, we suggest that this subset may have been a mixture of patients with LS, whose germline variants were not detected, and those with sporadic CRC. Future advances in NGS techniques may allow obtaining more accurate genetic information for discriminating between patients with LS and LLS.

Among the variants identified in this study, the largest category was VUS in MMR genes. The classification of these patients is still uncertain, and they were grouped as patients with LLS in the current study. Some of the patients carrying VUS in MMR genes may have been true patients with LS, which was supported by a high frequency of metachronous CRC. The pathogenicity of these VUS should be confirmed in functional experiments. The high frequency of metachronous CRC observed in our study suggests that patients with LLS should be considered high-risk cases, and strategies for cancer prevention must be implemented for this group of patients and their relatives. In clinical practice, aggressive surgical protocols, such as extended colectomy and subtotal colectomy, should be recommended. Platinum-based chemotherapy should be routinely applied, and treatment with immune checkpoint inhibitors may provide a considerable benefit to patients with metastatic MMR-deficient CRC. Meanwhile, stringent colonoscopy surveillance should be performed in patients with LLS, who carry both PVs and VUS in MMR genes.

In addition to MMR genes, most of the other PVs and likely PVs were detected in the MUTYH and GJB2 genes. Biallelic MUTYH variants have been detected in $1.8-3.1 \%$ of patients with LLS $[20,21]$. MUTYH associated polyposis is extremely variable, ranging from severe polyposis coli to attenuated forms with a late age of onset or few adenomas, or CRC, which creates a phenotypic overlap with LS [20, 22]. GJB2 encodes a gap junction protein, also known as connexin 26 . Variants in this gene are responsible for as much as $50 \%$ of prelingual, recessive deafness [23]. The cytoplasmic Cx26 protein has been associated with the tumor progression and a poor prognosis in patients with breast cancer and esophageal squamous cell carcinoma $[24,25]$. To the best of our knowledge, this is the first study to demonstrate the involvement of $G J B 2$, as a novel candidate gene, in LLS-linked CRC. The pathogenicity of the frameshift variant in GJB2 is being evaluated by functional analysis, and the results will be reported separately. Variants in the 
exonuclease domain of the polymerase proofreading genes POLE and POLD1 cause polymerase proofreading-associated polyposis, which is a dominant-inheritance and high-penetrance hereditary syndrome conferring a predisposition to attenuated colorectal polyposis and early-onset CRC [26]. The association between variants of polymerase proofreading genes and $M M R$ deficiency has been reported previously [27]. In our study, VUS in the POLE and POLD1 genes were predicted to be deleterious and were among the most frequently detected variants. Some other variants were identified in $B R C A 1, B R C A 2$, and $R A D 50$, which are involved in the homologous recombination pathway. Defects in the BRCA genes are known to be pathogenic causes of hereditary breast and ovarian cancers [28], in addition to conferring a high risk of developing CRC [29].

Therefore, it is possible that some cases of LLS can be due to the pleiotropism of certain gene variants, manifesting as genetic overlaps with other hereditary cancer syndromes. Because of the mixture, a higher prevalence of extra-CRCs and a lower prevalence of CRCs were revealed in the LLS families. The high risk of extra-CRCs found in our study suggests that stringent surveillance of other organs should be recommended for probands and their affected family members. The surveillance regimen can be based on the gene variants and family history. For example, gastroduodenoscopy should be regularly performed in patients carrying MUTYH variants, while gynecological and breast examinations would be recommended for patients carrying $B R C A$ variants. Furthermore, functional analysis of the undefined variants found in patients with LLS should be performed to elucidate the underlying molecular etiology of LLS.

The difference in the age at onset of CRC between patients with LS and LLS remains controversial; some studies demonstrated similar proportions of early-onset patients in the LS and LLS groups [13], whereas one report showed that the population of patients with LLS was older [30]. Our results supported the latter findings, with age differences being manifested in both probands and related family members. Variants in genes such as POLE and BRCA, which were found in patients with LLS, may confer a higher risk of CRC; however, these variants show moderate penetrance [31]. Because sporadic CRC is combined with moderate penetrance of other variants, a delayed onset of CRC was demonstrated in probands with LLS. It is noteworthy that more than half of the patients in the LLS group were early-onset cases, which is significantly higher than the reported rate of sporadic CRC [32]. Therefore, MSI and multigene panel testing should be recommended for the early-onset subset, and screening colonoscopy at an early age should be performed in affected family members.

In terms of the CRC localization, our study showed a striking clustering of tumors in the rectum of probands with LLS, indicating that the rectum as the preferred organ can be described as a clinical feature of LLSassociated CRC. A higher frequency of left colon cancer was consistent with the findings of our previous study, which investigated clinical features of LS in an Asian population [33]. While LS-associated CRC is characterized by mucinous differentiation [34], a reasonably lower proportion of mucinous tumors was observed in the LLS cohort in this study.

Another interesting finding was a larger number of male patients in LLS families. A higher prevalence of male patients in LS families was reported in a previous review [35], but has not been previously described in 
LLS families. This discovery of the sex-dependent tendency of disease in LLS families indicates that more attention should be paid to the screening and surveillance of male members.

There are some limitations of our study. First, because this was a retrospective study, the potential bias in the selection of subjects could not be eliminated. Second, MSI testing was not performed, which may have resulted in an incorrect interpretation of the molecular evidence. Lastly, the sample size needs to be increased, and a long-term follow-up is required.

\section{Conclusions}

In conclusion, LLS should be classified as a mixed entity, containing cases of true LS, other hereditary cancer syndromes, and sporadic CRC. The high risks of CRC and extra-CRCs, which were found in this study, suggest that aggressive surgical management should be performed for probands with LLS, and a stringent surveillance protocol, which involves regular follow-ups of the intestine and other organs, should be recommended for probands and affected family members. The surveillance regimen can be based on gene variants and the family history. The preference for CRC development in the rectum should be described as a clinical feature of LLS. A higher prevalence of male patients was discovered, for the first time, in LLS families; this indicates that more attention should be paid to the screening and surveillance of male members.

\section{Abbreviations}

\section{LS}

Lynch syndrome; CRC:colorectal cancer; MMR:mismatch repair; MSI:microsatellite instability; PV:pathogenic variants; LLS:Lynch-like syndrome; VUS:variants of unknown significance; NGS:Next-generation sequencing

\section{Declarations}

\section{Ethics approval and consent to participate}

All examinations and treatments were conducted at the Fudan University Shanghai Cancer Center (Shanghai, China) and were in accordance with the Declaration of Helsinki. This study was approved by the Ethics Committee of the Fudan University Shanghai Cancer Center. Written informed consent was obtained from all patients included in this study

\section{Consent for publication}

Not applicable.

\section{Availability of data and materials}

The datasets used and analyzed during the current study are available from the corresponding author on reasonable request. 


\section{Competing interests}

The authors declare that they have no competing interests.

\section{Funding}

Funding for this study was provided by the National Natural Science Foundation of China (NO. 81472620), Natural Science Foundation of Shanghai (NO. 16ZR1406700), Science and Technology Commission of Shanghai Municipality Foundation (NO. 18140903702) and the Development Foundation for Shanghai Talents (NO. 2017120). The funding source played no role in research design and collection, analysis, report writing, and the decision to submit articles for publication.

\section{Authors' contributions}

YX, YX and FQL conceived and designed the study. YX, CL, YQZ, and TAG collected and analyzed the datas. YX provided statistical expertise and were involved in data analysis and interpretation of results. $Y X$ wrote the paper. CL, YX and FQL reviewed the paper. All authors have read and approved the manuscript.

\section{Acknowledgements}

Not applicable.

\section{References}

1. Hampel H, Frankel WL, Martin E, Arnold M, Khanduja K, Kuebler P, Nakagawa H, Sotamaa K, Prior TW, Westman J, et al. Screening for the Lynch syndrome (hereditary nonpolyposis colorectal cancer). N Engl J Med. 2005;352(18):1851-60.

2. Tiwari AK, Roy HK, Lynch HT. Lynch syndrome in the 21st century: clinical perspectives. QJM. 2016;109(3):151-8.

3. Win AK, Buchanan DD, Rosty C, MacInnis RJ, Dowty JG, Dite GS, Giles GG, Southey MC, Young JP, Clendenning $\mathrm{M}$, et al. Role of tumour molecular and pathology features to estimate colorectal cancer risk for first-degree relatives. Gut. 2015;64(1):101-10.

4. Piñol V, Castells A, Andreu M, Castellví-Bel S, Alenda C, Llor X, Xicola RM, Rodríguez-Moranta F, Payá A, Jover R, et al. Accuracy of revised Bethesda guidelines, microsatellite instability, and immunohistochemistry for the identification of patients with hereditary nonpolyposis colorectal cancer. JAMA. 2005;293(16):1986-94.

5. Grady WM, Carethers JM. Genomic and epigenetic instability in colorectal cancer pathogenesis. Gastroenterology. 2008;135(4):1079-99.

6. Parsons MT, Buchanan DD, Thompson B, Young JP, Spurdle AB. Correlation of tumour BRAF mutations and $\mathrm{MLH} 1$ methylation with germline mismatch repair (MMR) gene mutation status: a literature review assessing utility of tumour features for MMR variant classification. J Med Genet. 2012 Mar;49(3):1517. 
7. Boland PM, Yurgelun MB, Boland CR. Recent progress in Lynch syndrome and other familial colorectal cancer syndromes. CA Cancer J Clin. 2018;68(3):217-31.

8. Rodríguez-Soler M, Pérez-Carbonell L, Guarinos C, Zapater P, Castillejo A, Barberá VM, Juárez M, Bessa $X$, Xicola RM, Clofent J, et al. Risk of cancer in cases of suspected lynch syndrome without germline mutation. Gastroenterology. 2013;144(5):926-32.

9. Carethers JM, Stoffel EM. Lynch syndrome and Lynch syndrome mimics: The growing complex landscape of hereditary colon cancer. World J Gastroenterol. 2015;21(31):9253-61.

10. Carethers JM. Differentiating Lynch-like from Lynch syndrome. Gastroenterology. 2014;146(3):602-4.

11. Katz LH, Burton-Chase AM, Advani S, Fellman B, Polivka KM, Yuan Y, Lynch PM, Peterson SK. Screening adherence and cancer risk perceptions in colorectal cancer survivors with Lynch-like syndrome. Clin Genet. 2016;89(3):392-8.

12. Woods MO, Younghusband HB, Parfrey PS, Gallinger S, McLaughlin J, Dicks E, Stuckless S, Pollett A, Bapat $B$, Mrkonjic $M$, et al. The genetic basis of colorectal cancer in a population-based incident cohort with a high rate of familial disease. Gut. 2010;59(10):1369-77.

13. Antelo M, Golubicki M, Roca E, Mendez G, Carballido M, Iseas S, Cuatrecasas M, Moreira L, Sanchez A, Carballal $S$, et al. Lynch-like syndrome is as frequent as Lynch syndrome in early-onset nonfamilial nonpolyposis colorectal cancer. Int J Cancer. 2019;145(3):705-13.

14. Gryfe R, Kim H, Hsieh ET, Aronson MD, Holowaty EJ, Bull SB, Redston M, Gallinger S. Tumor microsatellite instability and clinical outcome in young patients with colorectal cancer. N Engl J Med. 2000;342(2):69-77.

15. Ribic CM, Sargent DJ, Moore MJ, Thibodeau SN, French AJ, Goldberg RM, Hamilton SR, Laurent-Puig P, Gryfe R, Shepherd LE, et al. Tumor microsatellite-instability status as a predictor of benefit from fluorouracil-based adjuvant chemotherapy for colon cancer. N Engl J Med. 2003;349(3):247-57.

16. Le DT, Durham JN, Smith KN, Wang H, Bartlett BR, Aulakh LK, Lu S, Kemberling H, Wilt C, Luber BS, et al. Mismatch repair deficiency predicts response of solid tumors to PD-1 blockade. Science. 2017;357(6349):409-13.

17. Clendenning M, Buchanan DD, Walsh MD, Nagler B, Rosty C, Thompson B, Spurdle AB, Hopper JL, Jenkins MA, Young JP. Mutation deep within an intron of MSH2 causes Lynch syndrome. Fam Cancer. 2011;10(2):297-301.

18. Morak M, Koehler U, Schackert HK, Steinke V, Royer-Pokora B, Schulmann K, Kloor M, Höchter W, Weingart J, Keiling C, et al. Biallelic MLH1 SNP cDNA expression or constitutional promoter methylation can hide genomic rearrangements causing Lynch syndrome. J Med Genet. 2011;48(8):513-9.

19. Liu Q, Thompson BA, Ward RL, Hesson LB, Sloane MA. Understanding the pathogenicity of noncoding mismatch repair gene promoter variants in Lynch syndrome. Hum Mutat. 2016;37(5):417-26.

20. Morak M, Heidenreich B, Keller G, Hampel H, Laner A, de la Chapelle A, Holinski-Feder E. Biallelic MUTYH mutations can mimic Lynch syndrome. Eur J Hum Genet. 2014;22(11):1334-7.

21. Vargas-Parra GM, González-Acosta M, Thompson BA, Gómez C, Fernández A, Dámaso E, Pons T, Morak M, Del Valle J, Iglesias S, et al. Elucidating the molecular basis of MSH2-deficient tumors by combined germline and somatic analysis. Int J Cancer. 2017;141(7):1365-80. 
22. Morak M, Laner A, Bacher U, Keiling C, Holinski-Feder E. MUTYH-associated polyposis - variability of the clinical phenotype in patients with biallelic and monoallelic MUTYH mutations and report on novel mutations. Clin Genet. 2010;78(4):353-63.

23. Smith RJ, Bale JF Jr, White KR. Sensorineural hearing loss in children. Lancet. 2005;365(9462):879-90.

24. Naoi Y, Miyoshi Y, Taguchi T, Kim SJ, Arai T, Tamaki Y, et al. Connexin26 expression is associated with lymphatic vessel invasion and poor prognosis in human breast cancer. Breast Cancer Res Treat. 2007;106:11-7.

25. Inose T, Kato H, Kimura H, Faried A, Tanaka N, Sakai M, Sano A, Sohda M, Nakajima M, Fukai Y, et al. Correlation between connexin 26 expression and poor prognosis of esophageal squamous cell carcinoma. Ann Surg Oncol. 2009;16(6):1704-10.

26. Palles C, Cazier JB, Howarth KM, Domingo E, Jones AM, Broderick P, Kemp Z, Spain SL, Guarino E, Salguero I, et al. Germline mutations affecting the proofreading domains of POLE and POLD1 predispose to colorectal adenomas and carcinomas. Nat Genet. 2013;45(2):136-44.

27. Jansen AM, van Wezel T, van den Akker BE, Ventayol Garcia M, Ruano D, Tops CM, Wagner A, Letteboer TG, Gómez-García EB, Devilee P, et al. Combined mismatch repair and POLE/POLD1 defects explain unresolved suspected Lynch syndrome cancers. Eur J Hum Genet. 2016;24(7):1089-92.

28. Llort G, Chirivella I, Morales R, Serrano R, Sanchez AB, Teulé A, Lastra E, Brunet J, Balmaña J, Graña B, SEOM Hereditary Cancer Working Group. SEOM clinical guidelines in Hereditary Breast and ovarian cancer. Clin Transl Oncol. 2015;17(12):956-61.

29. Mersch J, Jackson MA, Park M, Nebgen D, Peterson SK, Singletary C, Arun BK, Litton JK. Cancers associated with BRCA1 and BRCA2 mutations other than breast and ovarian. Cancer. 2015;121(2):26975.

30. Porkka N, Lahtinen L, Ahtiainen M, Böhm JP, Kuopio T, Eldfors S, Mecklin JP, Seppälä TT. Peltomäki P. Epidemiological, clinical and molecular characterization of Lynch-like syndrome: A population-based study. Int J Cancer. 2019;145(1):87-98.

31. Yurgelun MB, Kulke MH, Fuchs CS, Allen BA, Uno H, Hornick JL, Ukaegbu Cl, Brais LK, McNamara PG, Mayer RJ, et al. Cancer Susceptibility Gene Mutations in Individuals With Colorectal Cancer. J Clin Oncol. 2017;35(10):1086-95.

32. Siegel RL, Miller KD, Fedewa SA, Ahnen DJ, Meester RGS, Barzi A, Jemal A. Colorectal cancer statistics, 2017. CA Cancer J Clin. 2017;67(3):177-93.

33. Liu F, Yang L, Zhou X, Sheng W, Cai S, Liu L, Nan P, Xu Y. Clinicopathological and genetic features of Chinese hereditary nonpolyposis colorectal cancer (HNPCC). Med Oncol. 2014(10):223.

34. Llor X, Pons E, Xicola RM, Castells A, Alenda C, Piñol V, Andreu M, Castellví-Bel S, Payá A, Jover R, et al. Differential features of colorectal cancers fulfilling Amsterdam criteria without involvement of the mutator pathway. Clin Cancer Res. 2005;11(20):7304-10.

35. Sehgal R, Sheahan K, O'Connell PR, Hanly AM, Martin ST, Winter DC. Lynch syndrome: an updated review. Genes (Basel). 2014;5(3):497-507. 